\title{
ON THE TIME SPENT IN THE RED BY A REFRACTED LÉVY RISK PROCESS
}

\author{
JEAN-FRANÇOIS RENAUD, ${ }^{*}$ UQAM
}

\begin{abstract}
In this paper we introduce an insurance ruin model with an adaptive premium rate, henceforth referred to as restructuring/refraction, in which classical ruin and bankruptcy are distinguished. In this model the premium rate is increased as soon as the wealth process falls into the red zone and is brought back to its regular level when the wealth process recovers. The analysis is focused mainly on the time a refracted Lévy risk process spends in the red zone (analogous to the duration of the negative surplus). Building on results from [11] and [16], we identify the distribution of various functionals related to occupation times of refracted spectrally negative Lévy processes. For example, these results are used to compute both the probability of bankruptcy and the probability of Parisian ruin in this model with restructuring.
\end{abstract}

Keywords: Spectrally negative Lévy process; refraction; occupation time; bankruptcy; Parisian ruin

2010 Mathematics Subject Classification: Primary 60G51

Secondary 91B30

\section{Introduction}

In classical actuarial ruin theory the time of default is assumed to occur if and when the surplus process falls below a certain threshold level for the first time. Without loss of generality, which is due to the spatial homogeneity of most surplus processes, this threshold level has commonly been assumed to be the artificial level 0 . For solvency purposes it is more appropriate to view this threshold level as the insurer's solvency capital requirement (SCR), which is set by the regulatory body. Therefore, new risk concepts and models have recently been introduced: Parisian ruin (see, for example, [5], [14], and [15]), random observations (see, for example, [2] and [3]) and Omega models (see, for example, [1], [4], and [7]).

Our goal is to introduce an insurance ruin model where default and bankruptcy are disentangled, and where restructuring is also considered. Indeed, it seems very likely that when the company is in financial distress, namely when the surplus process falls below the critical level (SCR), some sort of restructuring will be undertaken. We propose a model with an adaptive premium rate, i.e. where the premium is increased as soon as the surplus process is in the so-called red zone and then returned to its regular level when the surplus process is out of the red zone. It is assumed that this critical situation is due to temporarily bad luck. Therefore, to achieve this we will use a refracted Lévy risk process, previously studied by Kyprianou and Loeffen [11], as our surplus process.

In conclusion, we propose to study the different occupation time related definitions of bankruptcy/default in a Lévy risk model with restructuring (a refracted Lévy risk model).

Received 28 August 2013; revision received 20 December 2013.

* Postal address: Département de Mathématiques, Université du Québec à Montréal (UQAM), 201 Av. PrésidentKennedy, Montréal, Québec, H2X 3Y7, Canada. Email address: renaud.jf@uqam.ca 
Despite the generality of using a Lévy process as the underlying surplus process, our model is very tractable thanks to the work of Kyprianou and Loeffen [11] and the fluctuation identities they have obtained. Traditionally, this type of risk process has been used to build models with a constant threshold dividend strategy; see the references in [11] and [12]. Recently, a number of identities concerned with the distribution of occupation times until first passage times for a refracted Lévy process were obtained [13]. Instead of borrowing results directly from [13], we will use results and techniques from [16] to derive (more general) results expressed solely in terms of the scale functions of the underlying process.

As a consequence, our new identities for the distribution of occupation times of refracted Lévy processes could form the theoretical basis for further study to develop a set of risk measures for this Lévy risk model with restructuring/refraction.

\subsection{The model}

Let $U$ be the surplus process of interest. We choose the level $b>0$ to be the threshold level representing the insurer's solvency capital requirement. As soon as $U$ goes below this critical level, restructuring will be undertaken in the sense that the premium for large claims will be (temporarily) increased. When $U$ recovers, that is, when $U$ goes above $b$ again, then things return to their initial state. In other words, we consider the level $b$ to be the critical level and the interval $(-\infty, b)$ to be the red zone. When the surplus process $U$ falls below $b$, a restructuring of the business is undertaken and this materializes itself by an increase in the drift of the process.

Intuitively, we are interested in the following dynamic:

$$
\mathrm{d} U_{t}=\mathrm{d} Y_{t}+\alpha \mathbf{1}_{\left\{U_{t}<b\right\}} \mathrm{d} t \quad \text { or, equivalently, } \quad U_{t}=Y_{t}+\alpha \int_{0}^{t} \mathbf{1}_{\left\{U_{s}<b\right\}} \mathrm{d} s,
$$

where $Y$ is the underlying (uncontrolled) risk process during standard business periods. However, for ease of presentation, namely to make our paper closer in notation to [11], we will use instead the following equivalent point of view. However, for ease of presentation, namely to make our paper closer in notation to [11], we will instead use the following equivalent point of view: we first set the dynamic of the surplus process in the red zone and refract it when it goes above $b$. Mathematically, let $X$ be the risk process during periods of financial distress, and define $U$ as

$$
\mathrm{d} U_{t}=\mathrm{d} X_{t}-\alpha \mathbf{1}_{\left\{U_{t}>b\right\}} \mathrm{d} t .
$$

For example, if $X$ is of the form

$$
X_{t}=\mathrm{c} t-S_{t}
$$

where c represents the premium rate and where the driftless subordinator $S=\left(S_{t}\right)_{t \geq 0}$ represents the aggregate claim payments, then $U$ has a drift value of $\mathrm{c}$ in the red zone (below $b$ ) and a drift value of $\mathrm{c}-\alpha$ above $b$. This includes the Cramér-Lundberg risk process as a special case. To avoid trivialities, we will assume that $\mathrm{c}>\alpha$ when $X$ has paths of bounded variation (see Condition (1)).

Our main result gives representations for the joint Laplace transforms of

$$
\left(\kappa_{a}^{-}, \int_{0}^{\kappa_{a}^{-}} \mathbf{1}_{\left\{U_{s}<b\right\}} \mathrm{d} s\right) \text { and }\left(\kappa_{c}^{+}, \int_{0}^{\kappa_{c}^{+}} \mathbf{1}_{\left\{U_{s}<b\right\}} \mathrm{d} s\right),
$$


where $a \leq x, b \leq c$, and where $\kappa_{a}^{-}$and $\kappa_{c}^{+}$are first passage times, in terms of the so-called scale functions of the underlying Lévy processes $X$ and $Y$. These quantities will then be used to study the probability of bankruptcy and the probability of Parisian ruin for $U$.

The rest of the paper is organized as follows. In Section 2 we introduce spectrally negative Lévy processes and refracted Lévy processes, including useful identities involving scale functions. In Section 3 we present the main result of the paper, while in Section 4 and Section 5 corollaries are derived and applied to the computations of the probability of bankruptcy and the probability of Parisian ruin, respectively.

\section{Spectrally negative Lévy processes}

On the filtered probability space $\left(\Omega, \mathcal{F},\left(\mathcal{F}_{t}\right)_{t \geq 0}, \mathbb{P}\right)$, let $X=\left(X_{t}\right)_{t \geq 0}$ be a spectrally negative Lévy process (SNLP), that is, a process with stationary and independent increments and no positive jumps. Hereby we exclude the case that $X$ is the negative of a subordinator, i.e. we exclude the case of $X$ having decreasing paths. The law of $X$ such that $X_{0}=x$ is denoted by $\mathbb{P}_{x}$ and the corresponding expectation by $\mathbb{E}_{x}$. We write $\mathbb{P}$ and $\mathbb{E}$ when $x=0$. As the Lévy process $X$ has no positive jumps, its Laplace transform exists: for $\lambda, t \geq 0$,

$$
\mathbb{E}\left[\mathrm{e}^{\lambda X_{t}}\right]=\mathrm{e}^{t \psi(\lambda)}
$$

where

$$
\psi(\lambda)=\gamma \lambda+\frac{1}{2} \sigma^{2} \lambda^{2}+\int_{0}^{\infty}\left(\mathrm{e}^{-\lambda z}-1+\lambda z \mathbf{1}_{(0,1]}(z)\right) \Pi(\mathrm{d} z)
$$

for $\gamma \in \mathbb{R}$ and $\sigma \geq 0$, and where $\Pi$ is a $\sigma$-finite measure on $(0, \infty)$ such that

$$
\int_{0}^{\infty}\left(1 \wedge z^{2}\right) \Pi(\mathrm{d} z)<\infty .
$$

This measure is called the Lévy measure of $X$, while $(\gamma, \sigma, \Pi)$ is referred to as the Lévy triplet of $X$. Note that, for convenience, we define the Lévy measure in such a way that it is a measure on the positive half-line instead of the negative half-line. Furthermore, note that $\mathbb{E}\left[X_{1}\right]=\psi^{\prime}(0+)$.

The process $X$ has paths of bounded variation if and only if $\sigma=0$ and $\int_{0}^{1} z \Pi(\mathrm{d} z)<\infty$. In this case we denote by $\mathrm{c}:=\gamma+\int_{0}^{1} z \Pi(\mathrm{d} z)>0$ the so-called drift of $X$ which can now be written as

$$
X_{t}=\mathrm{c} t-S_{t},
$$

where $S=\left(S_{t}\right)_{t \geq 0}$ is a driftless subordinator (for example, a gamma process or a compound Poisson process with positive jumps). When $S$ is a compound Poisson process and $\mathbb{E}\left[X_{1}\right]>0$ (net profit condition), we recover the classical Cramér-Lundberg risk process. Finally, if $\Pi(\mathrm{d} z) \equiv 0$, we recover the Brownian motion risk process, i.e. $X$ can then be written as

$$
X_{t}=\mathrm{c} t+\sigma B_{t},
$$

since $\mathrm{c}=\gamma$, where $B=\left(B_{t}\right)_{t \geq 0}$ is a standard Brownian motion. In the actuarial risk theory literature SNLPs have been referred to as general Lévy insurance risk processes.

\subsection{Scale functions and fluctuation identities}

For an arbitrary SNLP, the Laplace exponent $\psi$ is strictly convex and $\lim _{\lambda \rightarrow \infty} \psi(\lambda)=\infty$. Thus, there exists a function $\Phi:[0, \infty) \rightarrow[0, \infty)$ defined by $\Phi(q)=\sup \{\lambda \geq 0 \mid \psi(\lambda)=q\}$ (its right-inverse) such that

$$
\psi(\Phi(q))=q, \quad q \geq 0 .
$$


We have $\Phi(q)=0$ if and only if $q=0$ and $\psi^{\prime}(0+) \geq 0$.

We now recall the definition of the $q$-scale function $W^{(q)}$. For $q \geq 0$, the $q$-scale function of the process $X$ is defined as the continuous function with Laplace transform

$$
\int_{0}^{\infty} \mathrm{e}^{-\lambda y} W^{(q)}(y) \mathrm{d} y=\frac{1}{\psi(\lambda)-q} \text { for } \lambda>\Phi(q) .
$$

This function is unique, positive, and strictly increasing for $x \geq 0$. We extend $W^{(q)}$ to the whole real line by setting $W^{(q)}(x)=0$ for $x<0$. We write $W=W^{(0)}$ when $q=0$. The initial value of $W^{(q)}$ is known to be

$$
W^{(q)}(0)= \begin{cases}\frac{1}{\mathrm{c}} & \text { when } \sigma=0 \text { and } \int_{0}^{1} z \Pi(\mathrm{d} z)<\infty \\ 0 & \text { otherwise }\end{cases}
$$

where we used the following definition: $W^{(q)}(0)=\lim _{x \downarrow 0} W^{(q)}(x)$. We will also frequently use the following function:

$$
Z^{(q)}(x)=1+q \int_{0}^{x} W^{(q)}(y) \mathrm{d} y, \quad x \in \mathbb{R} .
$$

Fix $\alpha \geq 0$ and define $Y=\left(Y_{t}\right)_{t \geq 0}$ by $Y_{t}=X_{t}-\alpha t$. As we mentioned in the introduction, if $X$ has paths of bounded variation then it is assumed that

$$
0 \leq \alpha<\mathrm{c}=\gamma+\int_{(0,1)} z \Pi(\mathrm{d} z) .
$$

This condition is very intuitive and will be assumed throughout this paper. Indeed, recall that when the process $X$ has paths of bounded variation then we can write $X_{t}=\mathrm{c} t-S_{t}$, where $S=\left(S_{t}\right)_{t \geq 0}$ is a driftless subordinator. Condition (1) says that we do not want to remove all the drift. In general, when $X$ is a general spectrally negative Lévy risk process, we would typically want $U$ to visit $(b, \infty)$ with positive probability (when starting below level $b$ ), which amounts to $\mathbb{E}\left[X_{1}\right]>-\infty$; however, this condition is not needed for our main results.

Clearly, $Y$ is also a spectrally negative Lévy process. In fact, it has the same Gaussian coefficient $\sigma$ and Lévy measure $\Pi$ as $X$. Its Laplace exponent is given by $\lambda \mapsto \psi(\lambda)-\alpha \lambda$. The law of $Y$ such that $Y_{0}=y$ is denoted by $\boldsymbol{P}_{y}$ and the corresponding expectation by $\boldsymbol{E}_{y}$. For each $q \geq 0$, we will write $\mathbb{W}^{(q)}$ and $\mathbb{Z}^{(q)}$ for the scale functions associated with $Y$. Again, we write $\mathbb{W}=\mathbb{W}^{(q)}$ when $q=0$.

Now, for any $a, c \in \mathbb{R}$, we define the following stopping times:

$$
\begin{array}{ll}
\tau_{a}^{-}=\inf \left\{t>0: X_{t}<a\right\} & \text { and } \quad \tau_{c}^{+}=\inf \left\{t>0: X_{t}>c\right\}, \\
\nu_{a}^{-}=\inf \left\{t>0: Y_{t}<a\right\} & \text { and } \quad v_{c}^{+}=\inf \left\{t>0: Y_{t}>c\right\},
\end{array}
$$

with the convention inf $\varnothing=\infty$.

It is well known that if $a \leq x \leq c$ then the solution to the two-sided exit problem for $X$ is given by

$$
\begin{gathered}
\mathbb{E}_{x}\left[\mathrm{e}^{-q \tau_{c}^{+}} ; \tau_{c}^{+}<\tau_{a}^{-}\right]=\frac{W^{(q)}(x-a)}{W^{(q)}(c-a)}, \\
\mathbb{E}_{x}\left[\mathrm{e}^{-q \tau_{a}^{-}} ; \tau_{a}^{-}<\tau_{c}^{+}\right]=Z^{(q)}(x-a)-\frac{Z^{(q)}(c-a)}{W^{(q)}(c-a)} W^{(q)}(x-a),
\end{gathered}
$$


where, for a random variable $\Theta$ and an event $A, \mathbb{E}[\Theta ; A]:=\mathbb{E}\left[\Theta \mathbf{1}_{A}\right]$. Finally, in general, the classical probability of ruin is given by

$$
\mathbb{P}_{x}\left(\tau_{0}^{-}<\infty\right)=1-\left(\mathbb{E}\left[X_{1}\right] \vee 0\right) W(x)
$$

Of course, the same results hold for $Y$; for example,

$$
\boldsymbol{E}_{x}\left[\mathrm{e}^{-q v_{c}^{+}} ; v_{c}^{+}<v_{a}^{-}\right]=\frac{\mathbb{W}^{(q)}(x-a)}{\mathbb{W}^{(q)}(c-a)} .
$$

\subsection{Refracted Lévy processes}

Fix $b>0$ and consider the following stochastic differential equation:

$$
\mathrm{d} U_{t}=\mathrm{d} X_{t}-\alpha \mathbf{1}_{\left\{U_{t}>b\right\}} \mathrm{d} t, \quad t \geq 0 .
$$

Theorem 1. (Kyprianou and Loeffen [11].) For a fixed $X_{0}=x \in \mathbb{R}$, there exists a unique strong solution $U=\left(U_{t}\right)_{t \geq 0}$ to (5). Moreover, $U$ is a strong Markov process.

We now present fluctuation identities for refracted processes. First, we define the following functions related to $U$ (and to the scale functions of the underlying SNLPs $X$ and $Y$ ). For $x, a \in \mathbb{R}$ and $q \geq 0$, define

$$
\begin{aligned}
& w^{(q)}(x ; a)=W^{(q)}(x-a)+\alpha \mathbf{1}_{\{x \geq b\}} \int_{b}^{x} \mathbb{W}^{(q)}(x-y) W^{(q) \prime}(y-a) \mathrm{d} y, \\
& z^{(q)}(x ; a)=Z^{(q)}(x-a)+\alpha q \mathbf{1}_{\{x \geq b\}} \int_{b}^{x} \mathbb{W}^{(q)}(x-y) W^{(q)}(y-a) \mathrm{d} y .
\end{aligned}
$$

Note that when $x<b$,

$$
w^{(q)}(x ; a)=W^{(q)}(x-a) \text { and } z^{(q)}(x ; a)=Z^{(q)}(x-a) .
$$

Most of the notation follows [10].

As we will now see we could think of these functions as being the scale functions of the refracted process $U$. Firstly, for any $a, c \in \mathbb{R}$, we define the following stopping times:

$$
\kappa_{a}^{-}=\inf \left\{t>0: U_{t}<a\right\} \quad \text { and } \quad \kappa_{c}^{+}=\inf \left\{t>0: U_{t}>c\right\} .
$$

The next result provides a solution to the two-sided exit problem for $U$. It is essentially a restatement of Theorem 4 in [11] (see also [10]) and it generalizes the expressions in (2.1) and (3) corresponding to the case where $\alpha=0$.

Theorem 2. (Kyprianou and Loeffen [11].) For $q \geq 0$ and $a \leq x, b \leq c$, we have

$$
\mathbb{E}_{x}\left[\mathrm{e}^{-q \kappa_{c}^{+}} ; \kappa_{c}^{+}<\kappa_{a}^{-}\right]=\frac{w^{(q)}(x ; a)}{w^{(q)}(c ; a)}
$$

and

$$
\mathbb{E}_{x}\left[\mathrm{e}^{-q \kappa_{a}^{-}} ; \kappa_{a}^{-}<\kappa_{c}^{+}\right]=z^{(q)}(x ; a)-\frac{z^{(q)}(c ; a)}{w^{(q)}(c ; a)} w^{(q)}(x ; a) .
$$


Proof. The case when $a=0$ has been proved in [11]. For $a \in \mathbb{R}$ such that $a \leq x, b \leq c$, we use a quasi-space homogeneity property of $U$ :

$$
\mathbb{E}_{x}\left[\mathrm{e}^{-q \kappa_{c}^{+}} ; \kappa_{c}^{+}<\kappa_{a}^{-}\right]=\mathbb{E}_{x-a}\left[\mathrm{e}^{-q \tilde{\kappa}_{c-a}^{+}} ; \tilde{\kappa}_{c-a}^{+}<\tilde{\kappa}_{0}^{-}\right],
$$

where $\tilde{\kappa}_{c-a}^{+}$and $\tilde{\kappa}_{0}^{-}$represent stopping times associated with the solution of

$$
U_{t}=X_{t}-\alpha \int_{0}^{t} \mathbf{1}_{\left\{U_{s}>b-a\right\}} \mathrm{d} s, \quad t \geq 0 .
$$

Using Theorem 4 in [11] and changing variables, we obtain

$$
\begin{aligned}
\mathbb{E}_{x-a}\left[\mathrm{e}^{-q \tilde{\kappa}_{c-a}^{+}} ; \tilde{\kappa}_{c-a}^{+}<\tilde{\kappa}_{0}^{-}\right]= & W^{(q)}(x-a)+\alpha \mathbf{1}_{\{x-a \geq b-a\}} \\
& \times \int_{b-a}^{x-a} \mathbb{W}^{(q)}(x-a-y) W^{(q) \prime}(y) \mathrm{d} y \\
= & W^{(q)}(x-a)+\alpha \mathbf{1}_{\{x \geq b\}} \int_{b}^{x} \mathbb{W}^{(q)}(x-y) W^{(q) \prime}(y-a) \mathrm{d} y
\end{aligned}
$$

and the result follows, concluding the proof. The second identity is derived in the same way.

Finally, the probability of classical ruin for a refracted Lévy process is given by

$$
\mathbb{P}_{x}\left(\kappa_{0}^{-}<\infty\right)=1-\left(\frac{\boldsymbol{E}\left[Y_{1}\right] \vee 0}{1-\alpha W(b)}\right) w^{(0)}(x ; 0),
$$

where $\boldsymbol{E}\left[Y_{1}\right]=\mathbb{E}\left[X_{1}\right]-\alpha$. When the net profit condition is satisfied, which in this model amounts to $\alpha<\mathbb{E}\left[X_{1}\right]$, we have

$$
\begin{aligned}
\mathbb{P}_{x}\left(\kappa_{0}^{-}<\infty\right) & =1-\left(\frac{\mathbb{E}\left[X_{1}\right]-\alpha}{1-\alpha W(b)}\right) w^{(0)}(x ; 0) \\
& =1-\left(\frac{\mathbb{E}\left[X_{1}\right]-\alpha}{1-\alpha W(b)}\right)\left\{W(x)+\alpha \mathbf{1}_{\{x \geq b\}} \int_{b}^{x} \mathbb{W}(x-y) W^{\prime}(y) \mathrm{d} y\right\}
\end{aligned}
$$

Remark 1. If no restructuring is undertaken, i.e. if $\alpha=0$ then $U=X=Y$ and there is only one process in the model. Then, the probability of ruin is the one given in (4).

Remark 2. It is important to note that $U$, and therefore $\left\{w^{(q)}, q \geq 0\right\}$ and $\left\{z^{(q)}, q \geq 0\right\}$, all depend on the fixed values of $\alpha$ and $b$.

Before stating the main results of this paper, we present a few identities relating the different scale functions. We can show (by taking Laplace transforms on both sides of the next equation) that, for $p, q, x \geq 0$,

$$
\begin{aligned}
\alpha \int_{0}^{x} & \mathbb{W}^{(p)}(x-y) W^{(q)}(y) \mathrm{d} y+(p-q) \int_{0}^{x} \int_{0}^{y} \mathbb{W}^{(p)}(y-z) W^{(q)}(z) \mathrm{d} z \mathrm{~d} y \\
& =\int_{0}^{x} \mathbb{W}^{(p)}(y) \mathrm{d} y-\int_{0}^{x} W^{(q)}(y) \mathrm{d} y,
\end{aligned}
$$


which is a generalization of the first displayed equation in Section 8 of [11]. Differentiating with respect to $x$ yields the following identity:

$$
\begin{aligned}
& (q-p) \int_{0}^{x} \mathbb{W}^{(p)}(x-y) W^{(q)}(y) \mathrm{d} y \\
& \quad=W^{(q)}(x)-\mathbb{W}^{(p)}(x)+\alpha\left(W^{(q)}(0) \mathbb{W}^{(p)}(x)+\int_{0}^{x} \mathbb{W}^{(p)}(x-y) W^{(q) \prime}(y) \mathrm{d} y\right),
\end{aligned}
$$

which is a generalization of Equation (5) in [16]. Furthermore, we derive, for $x>b$,

$$
w^{(q)}(x ; 0)=\left(1-\alpha W^{(q)}(0)\right) \mathbb{W}^{(q)}(x)-\alpha \int_{0}^{b} \mathbb{W}^{(q)}(x-y) W^{(q) \prime}(y) \mathrm{d} y .
$$

\subsection{An example}

There are various examples of SNLPs for which an explicit formula exists for the scale function $W^{(q)}$. For example, when $X$ is a compound Poisson risk process with a jump distribution that has a Laplace transform which is the ratio of two polynomials, then the Laplace transform of the scale function $1 /(\psi(\lambda)-q)$ is also a rational function and an explicit expression for the scale function $W^{(q)}$ is known.

We now present the case of a Lévy jump-diffusion process where the jump distribution is a mixture of exponentials. In other words,

$$
X_{t}=\mathrm{c} t+\sigma B_{t}-\sum_{i=1}^{N_{t}} \xi_{i}
$$

where $\sigma>0, \mathrm{C} \in \mathbb{R}, B=\left(B_{t}\right)_{t \geq 0}$ is a Brownian motion, $N=\left(N_{t}\right)_{t \geq 0}$ is a Poisson process with intensity $\eta>0$, and $\left\{\xi_{1}, \xi_{2}, \ldots\right\}$ are independent, identically distributed and positive random variables with a common probability density function given by

$$
f_{\xi}(y)=\left(\sum_{i=1}^{n} a_{i} \alpha_{i} \mathrm{e}^{-\alpha_{i} y}\right) \mathbf{1}_{\{y>0\}},
$$

where $n$ is a positive integer, $0<\alpha_{1}<\alpha_{2}<\cdots<\alpha_{n}$, and $\sum_{i=1}^{n} a_{i}=1$, where $a_{i}>0$ for all $i=1, \ldots, n$. All of the aforementioned objects are mutually independent.

The Laplace exponent of $X$ is then clearly given by

$$
\psi(\lambda)=\mathrm{c} \lambda+\frac{1}{2} \sigma^{2} \lambda^{2}+\eta\left(\sum_{i=1}^{n} \frac{a_{i} \alpha_{i}}{\lambda+\alpha_{i}}-1\right)
$$

for $\lambda>-\alpha_{1}$. In this case,

$$
\mathbb{E}\left[X_{1}\right]=\psi^{\prime}(0+)=\mathrm{c}-\eta \sum_{i=1}^{n} \frac{a_{i}}{\alpha_{i}} .
$$

For $q>0$ or $\psi^{\prime}(0+) \neq 0$, we can write (see, for example, [16])

$$
\frac{1}{\psi(\lambda)-q}=\sum_{i=1}^{n+2}\left(\psi^{\prime}\left(\theta_{i}^{(q)}\right)\left(\lambda-\theta_{i}^{(q)}\right)\right)^{-1}
$$

for $\lambda \in \mathbb{R} \backslash\left(\left\{\theta_{1}^{(q)}, \ldots, \theta_{n+2}^{(q)}\right\} \cup\left\{\alpha_{1}, \ldots, \alpha_{n}\right\}\right)$, where $\theta_{1}^{(q)}>\theta_{2}^{(q)}>\cdots>\theta_{n+2}^{(q)}$ are the roots of $\lambda \mapsto \psi(\lambda)-q$. Note that $\theta_{1}^{(q)}=\Phi(q)$ and $\theta_{n+2}^{(q)}<-\alpha_{n}<\theta_{n+1}^{(q)}<-\alpha_{n-1}<\theta_{n}^{(q)} \cdots<$ $-\alpha_{1}<\theta_{2}^{(q)}<\theta_{1}^{(q)}$, since $\sigma$ is assumed here to be strictly positive. 
By Laplace inversion, we have for $q>0$ or for $q=0$ and $\psi^{\prime}(0) \neq 0$ that, for $x \geq 0$,

$$
\begin{aligned}
W^{(q)}(x) & =\sum_{i=1}^{n+2} \frac{\mathrm{e}^{\theta_{i}^{(q)} x}}{\psi^{\prime}\left(\theta_{i}^{(q)}\right)}, \\
Z^{(q)}(x) & = \begin{cases}q \sum_{i=1}^{n+2} \frac{\mathrm{e}^{\theta_{i}^{(q)} x}}{\psi^{\prime}\left(\theta_{i}^{(q)}\right) \theta_{i}^{(q)}} & \text { if } q>0, \\
1 & \text { if } q=0 .\end{cases}
\end{aligned}
$$

Of course, $\mathbb{W}^{(q)}$ and $\mathbb{Z}^{(q)}$ will look just the same (we only need to change $\mathrm{c}$ for $\mathrm{c}-\alpha$ at the beginning of the above procedure). Therefore, computing derivatives and integrals of those scale functions, in particular, expressions for $w^{(q)}$ and $z^{(q)}$, will be very easy thanks to the exponential form of all those scale functions.

Remark 3. We could also have phase-type distributed random variables, instead of a mixture of exponentials and still obtain scale functions that are the sum of exponential functions; see [6].

Moreover (when considering other examples for which the $q$-scale function is not known in explicit form), there are good numerical methods for dealing with Laplace inversion; [8, Section 5] deals specifically with Laplace inversion of the scale function.

Finally, the reader is referred to [9] and [10] for more details on spectrally negative Lévy processes and their use in ruin theory. For examples and numerical techniques related to the computation of scale functions, we suggest Kuznetsov et al. [8].

\section{Time spent in the red zone}

Now, we derive the joint Laplace transforms of

$$
\left(\kappa_{a}^{-}, \int_{0}^{\kappa_{a}^{-}} \mathbf{1}_{\left\{U_{s}<b\right\}} \mathrm{d} s\right) \text { and }\left(\kappa_{c}^{+}, \int_{0}^{\kappa_{c}^{+}} \mathbf{1}_{\left\{U_{s}<b\right\}} \mathrm{d} s\right),
$$

where $a \leq x, b \leq c$, from which all subsequent results will be derived. Note that, for example, the random variable $\int_{0}^{\kappa_{a}^{-}} \mathbf{1}_{\left\{U_{s}<b\right\}} \mathrm{d} s$ is the length of time spent by $U$ below $b$ (occupation time of the red zone) until level $a$ is crossed.

The structure of our main results is the same as in [16], thanks to the Markovian property of $U$ (see Theorem 1). Note that we limit ourselves to the time spent in the red zone, as opposed to any interval, overlapping or not with the red zone. However, our methodology applies to any finite interval, at the cost of a more complicated expression, involving extra convolution terms.

Theorem 3. It holds for $a \leq x, b \leq c$ and for $p, q \geq 0$,

$$
\begin{aligned}
\mathbb{E}_{x}\left[\mathrm{e}^{-p \kappa_{c}^{+}-q \int_{0}^{\kappa_{c}^{+}} \mathbf{1}_{\left\{U_{s}<b\right\}} \mathrm{d} s} ; \kappa_{c}^{+}<\kappa_{a}^{-}\right] \\
=\frac{w^{(p+q)}(x ; a)-q \int_{b}^{x} \mathbb{W}^{(p)}(x-y) w^{(p+q)}(y ; a) \mathrm{d} y}{w^{(p+q)}(c ; a)-q \int_{b}^{c} \mathbb{W}(p)(c-y) w^{(p+q)}(y ; a) \mathrm{d} y}
\end{aligned}
$$


and

$$
\begin{aligned}
\mathbb{E}_{x}\left[\mathrm{e}^{-p \kappa_{a}^{-}-q \int_{0}^{\kappa_{a}^{-}} \mathbf{1}_{\left\{U_{s}<b\right\}} \mathrm{d} s} ; \kappa_{a}^{-}<\kappa_{c}^{+}\right] \\
=z^{(p+q)}(x ; a)-q \int_{b}^{x} \mathbb{W}^{(p)}(x-y) z^{(p+q)}(y ; a) \mathrm{d} y \\
\quad+\frac{z^{(p+q)}(c ; a)-q \int_{b}^{c} \mathbb{W}^{(p)}(c-y) z^{(p+q)}(y ; a) \mathrm{d} y}{w^{(p+q)}(c ; a)-q \int_{b}^{c} \mathbb{W}^{(p)}(c-y) w^{(p+q)}(y ; a) \mathrm{d} y} \\
\quad \times\left(w^{(p+q)}(x ; a)-q \int_{b}^{x} \mathbb{W}^{(p)}(x-y) w^{(p+q)}(y ; a) \mathrm{d} y\right) .
\end{aligned}
$$

These results are fundamental in developing a risk management toolkit based on occupation times as we will see in the next sections. For example, we will derive several results needed to compute the probability of bankruptcy and the probability of Parisian ruin in this model with restructuring/refraction. They are extensions of the results obtained in [16] for the case in which $\alpha=0$. Also, our results improve the results in [13] because we are dealing with the case in which $p>0$ and consider a general starting point $x$.

Before proving Theorem 3, we will need the following technical lemma.

\subsection{Technical lemma}

For $q \geq 0$, we can define $\mathcal{V}_{b}^{(q)}(Y)$ to be the function space associated with the spectrally negative Lévy process $Y$ consisting of positive and measurable functions $v^{(q)}: \mathbb{R} \rightarrow \mathbb{R}$, that satisfy

$$
\boldsymbol{E}_{x}\left[\mathrm{e}^{-q v_{b}^{-}} v^{(q)}\left(Y_{v_{b}^{-}}\right) ; v_{b}^{-}<v_{c}^{+}\right]=v^{(q)}(x)-\frac{\mathbb{W}^{(q)}(x-b)}{\mathbb{W}^{(q)}(c-b)} v^{(q)}(c)
$$

for all $x, c$ such that $b \leq x \leq c$. For example, $\mathbb{W}^{(q)}$ and $\mathbb{Z}^{(q)}$ are elements of $\mathcal{V}_{b}^{(q)}(Y)$, which is clearly a linear space.

Then, Lemma 2.1 in [16] states that if $v^{(q)}(\cdot)$ is sufficiently well behaved we have, for all $p \geq 0$ and $x, c$ such that $b \leq x \leq c$,

$$
\begin{aligned}
\boldsymbol{E}_{x}\left[\mathrm{e}^{-p v_{b}^{-}} v^{(q)}\left(Y_{v_{b}^{-}}\right) ; v_{b}^{-}<v_{c}^{+}\right] \\
=v^{(q)}(x)-(q-p) \int_{b}^{x} \mathbb{W}^{(p)}(x-y) v^{(q)}(y) \mathrm{d} y \\
\quad-\frac{\mathbb{W}^{(p)}(x-b)}{\mathbb{W}^{(p)}(c-b)}\left(v^{(q)}(c)-(q-p) \int_{b}^{c} \mathbb{W}^{(p)}(c-y) v^{(q)}(y) \mathrm{d} y\right) .
\end{aligned}
$$

The next result is a generalization of Theorem 16 in [11]. Recall that $W^{(q)}$ and $Z^{(q)}$ are the scale functions associated with $X$, while $\mathbb{W}^{(q)}$ and $\mathbb{Z}^{(q)}$ are those associated with $Y$.

Lemma 1. For all $p, q \geq 0$ and $x$, $c$ such that $b \leq x \leq c$,

$$
\begin{aligned}
\boldsymbol{E}_{x}\left[\mathrm{e}^{\left.-p v_{b}^{-} W^{(q)}\left(Y_{v_{b}^{-}}\right) ; v_{b}^{-}<v_{c}^{+}\right]=}\right. & w^{(q)}(x ; 0)-(q-p) \int_{b}^{x} \mathbb{W}^{(p)}(x-y) w^{(q)}(y ; 0) \mathrm{d} y \\
& -\frac{\mathbb{W}^{(p)}(x-b)}{\mathbb{W}^{(p)}(c-b)}\left(w^{(q)}(c ; 0)-(q-p)\right. \\
& \left.\times \int_{b}^{c} \mathbb{W}^{(p)}(c-y) w^{(q)}(y ; 0) \mathrm{d} y\right) .
\end{aligned}
$$


and

$$
\begin{aligned}
\boldsymbol{E}_{x}\left[\mathrm{e}^{-p v_{b}^{-}} Z^{(q)}\left(Y_{v_{b}^{-}}\right) ; v_{b}^{-}<v_{c}^{+}\right]= & z^{(q)}(x ; 0)-(q-p) \int_{b}^{x} \mathbb{W}^{(p)}(x-y) z^{(q)}(y ; 0) \mathrm{d} y \\
& -\frac{\mathbb{W}^{(p)}(x-b)}{\mathbb{W}^{(p)}(c-b)}\left(z^{(q)}(c ; 0)-(q-p)\right. \\
& \left.\times \int_{b}^{c} \mathbb{W}^{(p)}(c-y) z^{(q)}(y ; 0) \mathrm{d} y\right) .
\end{aligned}
$$

Proof. Since $\left\{Y_{t}, t<v_{b}^{-}\right\}$under $\boldsymbol{P}_{x}$ and $\left\{U_{t}, t<\kappa_{b}^{-}\right\}$under $\mathbb{P}_{x}$ have the same law when $x \geq b$, we have

$$
\boldsymbol{E}_{x}\left[\mathrm{e}^{-p v_{b}^{-}} W^{(q)}\left(Y_{v_{b}^{-}}\right) ; v_{b}^{-}<v_{c}^{+}\right]=\mathbb{E}_{x}\left[\mathrm{e}^{-q \kappa_{b}^{-}} W^{(q)}\left(U_{\kappa_{b}^{-}}\right) ; \kappa_{b}^{-}<\kappa_{c}^{+}\right]
$$

Consequently, according to Lemma 2.1 in [16], it suffices to show that

$$
\mathbb{E}_{x}\left[\mathrm{e}^{-q \kappa_{b}^{-}} w^{(q)}\left(U_{\kappa_{b}^{-}} ; 0\right) ; \kappa_{b}^{-}<\kappa_{c}^{+}\right]=w^{(q)}(x ; 0)-\frac{\mathbb{W}^{(q)}(x-b)}{\mathbb{W}^{(q)}(c-b)} w^{(q)}(c ; 0) .
$$

The latter is obtained using the strong Markov property of $U$ (see Theorem 1) and the solution to the two-sided exit problem for $U$ (see Theorem 2): indeed, for $b \leq x \leq c$, we can write

$$
\begin{aligned}
\frac{w^{(q)}(x ; 0)}{w^{(q)}(c ; 0)}= & \mathbb{E}_{x}\left[\mathrm{e}^{-q \kappa_{c}^{+}} ; \kappa_{c}^{+}<\kappa_{0}^{-}\right] \\
= & \mathbb{E}_{x}\left[\mathrm{e}^{-q \kappa_{c}^{+}} ; \kappa_{c}^{+}<\kappa_{b}^{-}\right]+\mathbb{E}_{x}\left[\mathrm{e}^{-q \kappa_{c}^{+}} ; \kappa_{b}^{-}<\kappa_{c}^{+}<\kappa_{0}^{-}\right] \\
= & \frac{\mathbb{W}^{(q)}(x-b)}{\mathbb{W}^{(q)}(c-b)}+\boldsymbol{E}_{x}\left[\mathrm{e}^{-q v_{b}^{-}} \mathbb{E}_{Y_{b}^{-}}\left[\mathrm{e}^{-q \kappa_{c}^{+}} ; \kappa_{c}^{+}<\kappa_{0}^{-}\right] ; v_{b}^{-}<v_{c}^{+}\right] \\
= & \frac{\mathbb{W}^{(q)}(x-b)}{\mathbb{W}^{(q)}(c-b)}+\mathbb{E}_{b}\left[\mathrm{e}^{-q \kappa_{c}^{+}} ; \kappa_{c}^{+}<\kappa_{0}^{-}\right] \\
& \times \boldsymbol{E}_{x}\left[\mathrm{e}^{-q v_{b}^{-}} \mathbb{E}_{Y_{v_{b}^{-}}}\left[\mathrm{e}^{-q \tau_{b}^{+}} ; \tau_{b}^{+}<\tau_{0}^{-}\right] ; v_{b}^{-}<v_{c}^{+}\right] \\
= & \frac{\mathbb{W}^{(q)}(x-b)}{\mathbb{W}^{(q)}(c-b)}+\mathbb{E}_{b}\left[\mathrm{e}^{-q \kappa_{c}^{+}} ; \kappa_{c}^{+}<\kappa_{0}^{-}\right] \boldsymbol{E}_{x}\left[\mathrm{e}^{-q v_{b}^{-}} \frac{W^{(q)}\left(Y_{v_{b}^{-}}\right)}{W^{(q)}(b)} ; v_{b}^{-}<v_{c}^{+}\right],
\end{aligned}
$$

where we used, again, that $\left\{Y_{t}, t<v_{b}^{-}\right\}$under $\boldsymbol{P}_{x}$ and $\left\{U_{t}, t<\kappa_{b}^{-}\right\}$under $\mathbb{P}_{x}$ have the same law when $x \geq b$, but also $\left\{X_{t}, t<\tau_{b}^{+}\right\}$and $\left\{U_{t}, t<\kappa_{b}^{+}\right\}$have the same law under $\mathbb{P}_{x}$ when $x \leq b$. From the definition of $w^{(q)}(\cdot ; 0)$, we know that $W^{(q)}(\cdot)$ and $w^{(q)}(\cdot ; 0)$ coincide on $(-\infty, b]$, so then we have $W^{(q)}\left(Y_{v_{b}^{-}}\right)=w^{(q)}\left(Y_{v_{b}^{-}} ; 0\right)$.

From the above calculations, it follows that $w^{(q)}(\cdot ; 0)$ satisfies Property (9) and, thus,

$$
w^{(q)}(\cdot ; 0) \in \mathcal{V}_{b}^{(q)}(Y) \text { for all } q, b \geq 0 .
$$

The result follows from Lemma 2.1 in [16].

Similarly, we can prove that

$$
z^{(q)}(\cdot ; 0) \in \mathcal{V}_{b}^{(q)}(Y) \text { for all } q, b \geq 0
$$




\subsection{Proof of Theorem 3}

Fix $a \leq b \leq c$ and $p, q \geq 0$. For $x \in[a, c]$, define

$$
v(x)=\mathbb{E}_{x}\left[\mathrm{e}^{-p \kappa_{c}^{+}-q \int_{0}^{\kappa_{c}^{+}} \mathbf{1}_{\left\{U_{s}<b\right\}} \mathrm{d} s} ; \kappa_{c}^{+}<\kappa_{a}^{-}\right] .
$$

Using the strong Markov property of $X$, the fact that $X$ is skip free upward and (2.1) we can write, for $a \leq x<b$,

$$
\begin{aligned}
v(x) & =v(b) \mathbb{E}_{x}\left[\mathrm{e}^{-(p+q) \kappa_{b}^{+}} ; \kappa_{b}^{+}<\kappa_{a}^{-}\right] \\
& =v(b) \mathbb{E}_{x}\left[\mathrm{e}^{-(p+q) \tau_{b}^{+}} ; \tau_{b}^{+}<\tau_{a}^{-}\right] \\
& =v(b) \frac{W^{(p+q)}(x-a)}{W^{(p+q)}(b-a)} .
\end{aligned}
$$

Similarly, for $b \leq x \leq c$, we have

$$
\begin{aligned}
v(x) & =\mathbb{E}_{x}\left[\mathrm{e}^{-p \kappa_{c}^{+}} ; \kappa_{c}^{+}<\kappa_{b}^{-}\right]+\mathbb{E}_{x}\left[\mathrm{e}^{-p \kappa_{b}^{-}} v\left(U_{\kappa_{b}^{-}}\right) ; \kappa_{b}^{-}<\kappa_{c}^{+}\right] \\
& =\frac{\mathbb{W}^{(p)}(x-b)}{\mathbb{W}^{(p)}(c-b)}+\frac{v(b)}{W^{(p+q)}(b-a)} \mathbb{E}_{x}\left[\mathrm{e}^{-p \kappa_{b}^{-}} W^{(p+q)}\left(U_{\kappa_{b}^{-}}-a\right) ; \kappa_{b}^{-}<\kappa_{c}^{+}\right] .
\end{aligned}
$$

We now assume that $X$ has paths of bounded variation. In this case we have $\mathbb{W}^{(p)}(0) \neq 0$ and, thus, setting $x=b$ in (10) yields

$$
v(b)=\frac{\mathbb{W}^{(p)}(0) / \mathbb{W}^{(p)}(c-b)}{1-\left(1 / W^{(p+q)}(b-a)\right) \mathbb{E}_{b}\left[\mathrm{e}^{-p \kappa_{b}^{-}} W^{(p+q)}\left(U_{\kappa_{b}^{-}}-a\right) ; \kappa_{b}^{-}<\kappa_{c}^{+}\right]} .
$$

Since $\left\{Y_{t}, t<v_{b}^{-}\right\}$under $\boldsymbol{P}_{x}$ and $\left\{U_{t}, t<\kappa_{b}^{-}\right\}$under $\mathbb{P}_{x}$ have the same law when $x \geq b$, and by spatial homogeneity of $Y$, we have

$$
\begin{aligned}
\mathbb{E}_{b}\left[\mathrm{e}^{-p \kappa_{b}^{-}} W^{(p+q)}\left(U_{\kappa_{b}^{-}}-a\right) ; \kappa_{b}^{-}<\kappa_{c}^{+}\right] \\
\quad=\boldsymbol{E}_{b}\left[\mathrm{e}^{-p v_{b}^{-}} W^{(p+q)}\left(Y_{v_{b}^{-}}-a\right) ; v_{b}^{-}<v_{c}^{+}\right] \\
\quad=\boldsymbol{E}_{b-a}\left[\mathrm{e}^{\left.-p v_{b-a}^{-} W^{(p+q)}\left(Y_{v_{b-a}^{-}}\right) ; v_{b-a}^{-}<v_{c-a}^{+}\right] .}\right.
\end{aligned}
$$

Using Lemma 1, we have

$$
\begin{aligned}
\mathbb{E}_{b}\left[\mathrm{e}^{-p \kappa_{b}^{-}} W^{(p+q)}\left(U_{\kappa_{b}^{-}}-a\right) ; \kappa_{b}^{-}<\kappa_{c}^{+}\right] \\
=w^{(p+q)}(b-a ; b-a)-\frac{\mathbb{W}^{(p)}(0)}{\mathbb{W}^{(p)}(c-b)} \\
\quad \times\left(w^{(p+q)}(c ; a)-q \int_{b}^{c} \mathbb{W}^{(p)}(c-y) w^{(p+q)}(y ; a) \mathrm{d} y\right),
\end{aligned}
$$

where $w^{(p+q)}(b-a ; b-a)=W^{(p+q)}(b-a)$. Substituting this into (11) yields

$$
v(b)=\frac{W^{(p+q)}(b-a)}{w^{(p+q)}(c ; a)-q \int_{b}^{c} \mathbb{W}(p)(c-y) w^{(p+q)}(y ; a) \mathrm{d} y} .
$$


Substituting the value of $v(b)$ just obtained into (10) and using Lemma 1 again yields,

$$
v(x)=\frac{w^{(p+q)}(x ; a)-q \int_{b}^{x} \mathbb{W}^{(p)}(x-y) w^{(p+q)}(y ; a) \mathrm{d} y}{w^{(p+q)}(c ; a)-q \int_{b}^{c} \mathbb{W}^{(p)}(c-y) w^{(p+q)}(y ; a) \mathrm{d} y} \quad \text { for } a \leq x \leq c .
$$

The case where $X$ has paths of unbounded variation follows using the same approximating procedure as in [16] (see also [13]).

The proof of the second part of the theorem follows similar lines and is omitted for brevity.

\section{Probability of bankruptcy}

We now apply our main result to compute the probability of bankruptcy. As mentioned previously, we consider level $b$ as a solvency capital requirement level and the interval $(-\infty, b)$ as the red zone. We choose the following definition for bankruptcy: if $U$ spends too much time in the red zone or if $U$ drops too deep, then bankruptcy is declared. To be more precise, for $q>0$, define the function $\omega: \mathbb{R} \rightarrow[0, \infty)$ by

$$
\omega(x)= \begin{cases}0 & \text { if } x \geq b \\ q & \text { if } 0 \leq x<b \\ \infty & \text { if } x<0,\end{cases}
$$

and the corresponding bankruptcy time $\rho_{\omega}$ by

$$
\rho_{\omega}=\inf \left\{t>0: \int_{0}^{t} \omega\left(U_{s}\right) \mathrm{d} s>\boldsymbol{e}_{1}\right\}
$$

where $\boldsymbol{e}_{1}$ is an independent exponentially distributed random variable with rate 1 . Therefore, bankruptcy occurs at rate $q$ when $U$ is between 0 and $b$, and bankruptcy occurs immediately if $U$ falls below level 0 . The choice of 0 as the ultimate acceptable surplus level is arbitrary and not restrictive.

Remark 4. This definition of bankruptcy is borrowed from that used in Omega models, in which the function $\omega$ is called the rate function. Typically, the rate function is chosen to be a decreasing function equalling zero above the critical level ( $b$ in our case) so that bankruptcy does not occur in this situation. This family of models was introduced in [4] and further investigated in [7] for Brownian motion with drift, in [16] for spectrally negative Lévy processes, and in [1] for compound Poisson processes and more general bankruptcy rate function. All these papers dealt with the case in which $\alpha=0$.

Suppose that the positive loading condition holds, which in our model means that $\mathbb{E}\left[X_{1}\right]>\alpha$. This implies that the probability of bankruptcy is less than one (see (6)). If bankruptcy does occur, either it occurs while the surplus is between 0 and $b$ or it occurs due to the surplus process dropping below level 0 . Mathematically, for any initial surplus $x \in \mathbb{R}$, we clearly have the following relationship:

$$
1=\mathbb{P}_{x}\left(0 \leq U_{\rho_{\omega}}<b, \rho_{\omega}<\infty\right)+\mathbb{P}_{x}\left(U_{\rho_{\omega}}<0, \rho_{\omega}<\infty\right)+\mathbb{P}_{x}\left(\rho_{\omega}=\infty\right)
$$


The probability that bankruptcy occurs while the surplus is between 0 and $b$ is given by

$$
\begin{aligned}
\mathbb{P}_{x}\left(0 \leq U_{\rho_{\omega}}<b, \rho_{\omega}<\infty\right) & =\mathbb{P}_{x}\left(\int_{0}^{\kappa_{0}^{-}} \omega\left(U_{s}\right) \mathrm{d} s>\boldsymbol{e}_{1}\right) \\
& =1-\mathbb{E}_{x}\left[\exp \left(-q \int_{0}^{\kappa_{0}^{-}} \mathbf{1}_{\left\{U_{s}<b\right\}} \mathrm{d} s\right)\right]
\end{aligned}
$$

Similarly, the probability that bankruptcy occurs due to the surplus process dropping below 0 is given by

$$
\begin{aligned}
\mathbb{P}_{x}\left(U_{\rho_{\omega}}<0, \rho_{\omega}<\infty\right) & =\mathbb{P}_{x}\left(\int_{0}^{\kappa_{0}^{-}} \omega\left(U_{s}\right) \mathrm{d} s \leq \boldsymbol{e}_{1}, \kappa_{0}^{-}<\infty\right) \\
& =\mathbb{E}_{x}\left[\exp \left(-q \int_{0}^{\kappa_{0}^{-}} \mathbf{1}_{\left\{U_{s}<b\right\}} \mathrm{d} s\right) ; \kappa_{0}^{-}<\infty\right]
\end{aligned}
$$

In conclusion, the answer is included in the following corollary.

Corollary 1. Assume the net profit condition $\mathbb{E}\left[X_{1}\right]>\alpha$ is verified.

(i) For $x, b, q \geq 0$,

$$
\begin{aligned}
\mathbb{E}_{x}\left[\exp \left(-q \int_{0}^{\kappa_{0}^{-}} \mathbf{1}_{\left\{U_{s}<b\right\}} \mathrm{d} s\right) ; \kappa_{0}^{-}<\infty\right] \\
=z^{(q)}(x ; 0)-q \int_{b}^{x} \mathbb{W}(x-y) z^{(q)}(y ; 0) \mathrm{d} y \\
\quad+\frac{\left(\mathbb{E}\left[X_{1}\right]-\alpha\right)+q \int_{0}^{b}\left(\mathbb{Z}^{(q)}(y)-\alpha W^{(q)}(y) \mathbb{Z}^{(q)}(b-y)\right) \mathrm{d} y}{Z^{(q)}(b)-\alpha W^{(q)}(b)} \\
\quad \times\left(w^{(q)}(x ; 0)-q \int_{b}^{x} \mathbb{W}(x-y) w^{(q)}(y ; 0) \mathrm{d} y\right) .
\end{aligned}
$$

(ii) For $x, b, q \geq 0$,

$$
\begin{aligned}
& \mathbb{E}_{x}\left[\exp \left(-q \int_{0}^{\kappa_{0}^{-}} \mathbf{1}_{\left\{U_{s}<b\right\}} \mathrm{d} s\right) ; \kappa_{0}^{-}=\infty\right] \\
& =\left(\mathbb{E}\left[X_{1}\right]-\alpha\right) \frac{w^{(q)}(x ; 0)-q \int_{b}^{x} \mathbb{W}(x-y) w^{(q)}(y ; 0) \mathrm{d} y}{Z^{(q)}(b)-\alpha W^{(q)}(b)}
\end{aligned}
$$

Proof. For part (i), clearly

$$
\begin{aligned}
& \mathbb{E}_{x}\left[\exp \left(-q \int_{0}^{\kappa_{0}^{-}} \mathbf{1}_{\left\{U_{s}<b\right\}} \mathrm{d} s\right) ; \kappa_{0}^{-}<\infty\right] \\
& \quad=\lim _{c \rightarrow \infty} \mathbb{E}_{x}\left[\exp \left(-q \int_{0}^{\kappa_{0}^{-}} \mathbf{1}_{\left\{U_{s}<b\right\}} \mathrm{d} s\right) ; \kappa_{0}^{-}<\kappa_{c}^{+}\right] .
\end{aligned}
$$


From Theorem 3, we can know that

$$
\begin{aligned}
\mathbb{E}_{x}\left[\exp \left(-q \int_{0}^{\kappa_{0}^{-}} \mathbf{1}_{\left\{U_{s}<b\right\}} \mathrm{d} s\right) ; \kappa_{0}^{-}<\kappa_{c}^{+}\right] \\
=z^{(q)}(x ; 0)-q \int_{b}^{x} \mathbb{W}(x-y) z^{(q)}(y ; 0) \mathrm{d} y \\
+\frac{z^{(q)}(c ; 0)-q \int_{b}^{c} \mathbb{W}(c-y) z^{(q)}(y ; 0) \mathrm{d} y}{w^{(q)}(c ; 0)-q \int_{b}^{c} \mathbb{W}(c-y) w^{(q)}(y ; 0) \mathrm{d} y} \\
\quad \times\left(w^{(q)}(x ; 0)-q \int_{b}^{x} \mathbb{W}(x-y) w^{(q)}(y ; 0) \mathrm{d} y\right) .
\end{aligned}
$$

We can show that, for $c>b$,

$$
\begin{aligned}
w^{(q)}(c ; 0)-q \int_{b}^{c} \mathbb{W}(c-y) w^{(q)}(y ; 0) \mathrm{d} y \\
=\left(1-\alpha W^{(q)}(0)\right)\left\{\mathbb{W}(c)+q \int_{0}^{b} \mathbb{W}(c-y) \mathbb{W}^{(q)}(y) \mathrm{d} y\right\} \\
\quad-\alpha \int_{0}^{b} W^{(q) \prime}(z)\left\{\mathbb{W}(c-z)+q \int_{0}^{b-z} \mathbb{W}(c-z-y) \mathbb{W}^{(q)}(y) \mathrm{d} y\right\} \mathrm{d} z .
\end{aligned}
$$

To prove this last identity, we used (7) and (8); the details are left to the reader. Therefore, since it is assumed that $\alpha<\mathbb{E}\left[X_{1}\right]$, we have $\lim _{c \rightarrow \infty} \mathbb{W}(c)=\left(\psi^{\prime}(0+)-\alpha\right)^{-1}=\left(\mathbb{E}\left[X_{1}\right]-\alpha\right)^{-1}$, and then we obtain (using the monotone convergence theorem)

$$
\begin{aligned}
\lim _{c \rightarrow \infty} & \frac{w^{(q)}(c ; 0)-q \int_{b}^{c} \mathbb{W}(c-y) w^{(q)}(y ; 0) \mathrm{d} y}{\mathbb{W}(c)} \\
& =\left(1-\alpha W^{(q)}(0)\right) \mathbb{Z}^{(q)}(b)-\alpha \int_{0}^{b} W^{(q) \prime}(z) \mathbb{Z}^{(q)}(b-z) \mathrm{d} z .
\end{aligned}
$$

Integrating by parts (or taking Laplace transforms on both sides) yields

$$
Z^{(q)}(x)-\alpha W^{(q)}(x)=\left(1-\alpha W^{(q)}(0)\right) \mathbb{Z}^{(q)}(x)-\alpha \int_{0}^{x} W^{(q) \prime}(y) \mathbb{Z}^{(q)}(x-y) \mathrm{d} y .
$$

We can also show that, for $c>b$,

$$
\begin{aligned}
z^{(q)}(c ; 0) & -q \int_{b}^{c} \mathbb{W}(c-y) z^{(q)}(y ; 0) \mathrm{d} y \\
= & 1+q \int_{0}^{b} \mathbb{W}(c-y) \mathbb{Z}^{(q)}(y) \mathrm{d} y \\
& -\alpha q \int_{0}^{b} W^{(q)}(z)\left\{\mathbb{W}(c-z)+q \int_{0}^{b-z} \mathbb{W}(c-z-y) \mathbb{W}^{(q)}(y) \mathrm{d} y\right\} \mathrm{d} z .
\end{aligned}
$$

Again, the details are left to the reader. Then, as above, we have

$$
\begin{aligned}
\lim _{c \rightarrow \infty} & \frac{z^{(q)}(c ; 0)-q \int_{b}^{c} \mathbb{W}(c-y) z^{(q)}(y ; 0) \mathrm{d} y}{\mathbb{W}(c)} \\
& =\left(\mathbb{E}\left[X_{1}\right]-\alpha\right)+q \int_{0}^{b}\left(\mathbb{Z}^{(q)}(y)-\alpha W^{(q)}(y) \mathbb{Z}^{(q)}(b-y)\right) \mathrm{d} y,
\end{aligned}
$$


and the result follows.

For part (ii), we also have

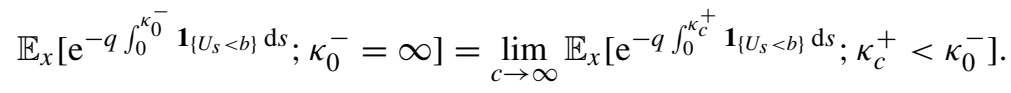

From Theorem 3, we know that

$$
\mathbb{E}_{x}\left[\mathrm{e}^{-q \int_{0}^{\kappa_{c}^{+}} \mathbf{1}_{\left\{U_{s}<b\right\}} \mathrm{d} s} ; \kappa_{c}^{+}<\kappa_{0}^{-}\right]=\frac{w^{(q)}(x ; 0)-q \int_{b}^{x} \mathbb{W}(x-y) w^{(q)}(y ; 0) \mathrm{d} y}{w^{(q)}(c ; 0)-q \int_{b}^{c} \mathbb{W}(c-y) w^{(q)}(y ; 0) \mathrm{d} y}
$$

and, by the above, the result follows.

\section{Probability of Parisian ruin}

A definition of Parisian ruin was proposed in [14]. In this definition of ruin, each excursion of the surplus process $U$ below the critical level $b$ is accompanied by an independent copy of an independent (of $U$ ) random variable. This is called the implementation clock. If the duration of a given excursion below $b$ is less than its associated implementation clock then Parisian ruin does not occur. We define the Parisian ruin time $\tau_{q}$ as the first time an implementation clock rings before the end of its corresponding excursion below $b$.

It can be shown that if the implementation clock is exponentially distributed with rate $q$ then the probability of Parisian ruin is given by

$$
\mathbb{P}_{x}\left(\tau_{q}<\infty\right)=1-\mathbb{E}_{x}\left[\exp \left(-q \int_{0}^{\infty} \mathbb{I}_{\left\{U_{s}<b\right\}} \mathrm{d} s\right)\right]
$$

when the net profit condition is verified. It turns out that this other definition of ruin is also related to Omega models.

Corollary 2. Assume the net profit condition $\mathbb{E}\left[X_{1}\right]>\alpha$ is verified.

(i) For $x, b \leq c$ and $q \geq 0$,

$$
\mathbb{E}_{x}\left[\mathrm{e}^{-q \int_{0}^{\kappa_{c}^{+}} \mathbf{1}_{\left\{U_{s}<b\right\}} \mathrm{d} s} ; \kappa_{c}^{+}<\infty\right]=\frac{\mathrm{e}^{\Phi(q)(x-b)}\left(1-(q-\alpha \Phi(q)) \int_{0}^{x-b} \mathrm{e}^{-\Phi(q) y} \mathbb{W}(y) \mathrm{d} y\right)}{\mathrm{e}^{\Phi(q)(c-b)}\left(1-(q-\alpha \Phi(q)) \int_{0}^{c-b} \mathrm{e}^{-\Phi(q) y} \mathbb{W}(y) \mathrm{d} y\right)} .
$$

(ii) For $x \in \mathbb{R}$ and $q \geq 0$,

$$
\begin{aligned}
& \mathbb{E}_{x}\left[\mathrm{e}^{-q \int_{0}^{\infty} \mathbf{1}_{\left\{U_{s}<b\right\}} \mathrm{d} s}\right] \\
& \quad=\left(\frac{\left(\mathbb{E}\left[X_{1}\right]-\alpha\right) \Phi(q)}{q-\alpha \Phi(q)}\right) \mathrm{e}^{\Phi(q)(x-b)}\left(1-(q-\alpha \Phi(q)) \int_{0}^{x-b} \mathrm{e}^{-\Phi(q) y} \mathbb{W}(y) \mathrm{d} y\right) .
\end{aligned}
$$

Moreover, we obtain the distribution

$$
\begin{aligned}
& \mathbb{P}_{x}\left(\int_{0}^{\infty} \mathbf{1}_{\left\{U_{s}<b\right\}} \mathrm{d} s \in \mathrm{d} r\right) \\
& \quad=\left(\mathbb{E}\left[X_{1}\right]-\alpha\right)\left\{\mathbb{W}(x-b) \delta_{0}(\mathrm{~d} r)+\int_{0}^{\infty} \frac{y}{r} \mathbb{W}^{\prime}(y+x-b) \mathbb{P}\left(X_{r} \in \mathrm{d} y\right) \mathrm{d} r\right\} .
\end{aligned}
$$


Proof. For part (i), clearly

$$
\mathbb{E}_{x}\left[\mathrm{e}^{-q \int_{0}^{\kappa_{c}^{+}}} \mathbf{1}_{\left\{U_{s}<b\right\}} \mathrm{d} s ; \kappa_{c}^{+}<\infty\right]=\lim _{m \rightarrow \infty} \mathbb{E}_{x}\left[\mathrm{e}^{-q \int_{0}^{\kappa_{c}^{+}} \mathbf{1}_{\left\{U_{s}<b\right\}} \mathrm{d} s} ; \kappa_{c}^{+}<\kappa_{-m}^{-}\right] .
$$

From Theorem 3, we have

$$
\mathbb{E}_{x}\left[\mathrm{e}^{-q \int_{0}^{\kappa_{c}^{+}} \mathbf{1}_{\left\{U_{s}<b\right\}} \mathrm{d} s} ; \kappa_{c}^{+}<\kappa_{-m}^{-}\right]=\frac{w^{(q)}(x ;-m)-q \int_{b}^{x} \mathbb{W}(x-y) w^{(q)}(y ;-m) \mathrm{d} y}{w^{(q)}(c ;-m)-q \int_{b}^{c} \mathbb{W}(c-y) w^{(q)}(y ;-m) \mathrm{d} y} .
$$

Since, for any $x$,

$$
\lim _{m \rightarrow \infty} \frac{W^{(q)}(x+m)}{W^{(q)}(m)}=\mathrm{e}^{\Phi(q) x} \quad \text { and } \quad \lim _{m \rightarrow \infty} \frac{W^{(q) \prime}(x+m)}{W^{(q)}(m)}=\Phi(q) \mathrm{e}^{\Phi(q) x},
$$

we obtain

$$
\lim _{m \rightarrow \infty} \frac{w^{(q)}(x ;-m)}{W^{(q)}(m)}=\mathrm{e}^{\Phi(q) x}+\alpha \Phi(q) \int_{b}^{x} \mathrm{e}^{\Phi(q) y} \mathbb{W}^{(q)}(x-y) \mathrm{d} y .
$$

Consequently,

$$
\begin{aligned}
\lim _{m \rightarrow \infty} & \frac{w^{(q)}(x ;-m)-q \int_{b}^{x} \mathbb{W}(x-y) w^{(q)}(y ;-m) \mathrm{d} y}{W^{(q)}(m)} \\
= & \mathrm{e}^{\Phi(q) x}+\alpha \Phi(q) \int_{b}^{x} \mathrm{e}^{\Phi(q) y} \mathbb{W}^{(q)}(x-y) \mathrm{d} y \\
& -q \int_{b}^{x} \mathbb{W}(x-y)\left(\mathrm{e}^{\Phi(q) y}+\alpha \Phi(q) \int_{b}^{y} \mathrm{e}^{\Phi(q) z} \mathbb{W}^{(q)}(y-z) \mathrm{d} z\right) \mathrm{d} y .
\end{aligned}
$$

Using (7) among other arguments, it can be shown that

$$
\begin{array}{rl}
\int_{b}^{x} & \mathbb{W}(x-y)\left(\mathrm{e}^{\Phi(q) y}+\alpha \Phi(q) \int_{b}^{y} \mathrm{e}^{\Phi(q) z} \mathbb{W}^{(q)}(y-z) \mathrm{d} z\right) \mathrm{d} y \\
\quad=\left(1-\frac{\alpha \Phi(q)}{q}\right) \int_{b}^{x} \mathrm{e}^{\Phi(q) y} \mathbb{W}(x-y) \mathrm{d} y+\left(\frac{\alpha \Phi(q)}{q}\right) \int_{b}^{x} \mathrm{e}^{\Phi(q) y} \mathbb{W}^{(q)}(x-y) \mathrm{d} y,
\end{array}
$$

and the result follows.

For part (ii), since the net profit condition is assumed, we have $\kappa_{c}^{+}<\infty$ almost surely for any $c$ and we have

$$
\lim _{x \rightarrow \infty} \mathbb{W}(x)=\left(\mathbb{E}\left[X_{1}\right]-\alpha\right)^{-1} .
$$

Therefore,

$$
\mathbb{E}_{x}\left[\mathrm{e}^{-q \int_{0}^{\infty} \mathbf{1}_{\left\{U_{s}<b\right\}} \mathrm{d} s}\right]=\lim _{c \rightarrow \infty} \mathbb{E}_{x}\left[\mathrm{e}^{-q \int_{0}^{\kappa_{c}^{+}} \mathbf{1}_{\left\{U_{s}<b\right\}} \mathrm{d} s} ; \kappa_{c}^{+}<\infty\right] .
$$

From the result in part (i), we have

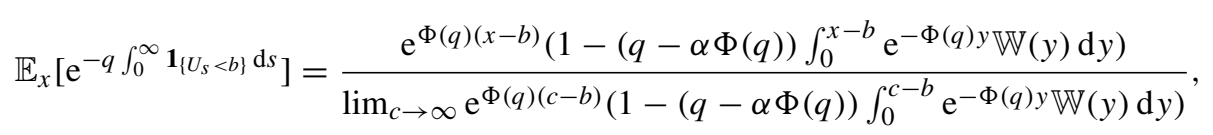


if the limit exists. In fact, for $c>b$, we can show that

$\mathrm{e}^{\Phi(q) c}\left(1-(q-\alpha \Phi(q)) \int_{0}^{c-b} \mathrm{e}^{-\Phi(q) y} \mathbb{W}(y) \mathrm{d} y\right)=(q-\alpha \Phi(q)) \int_{-b}^{\infty} \mathrm{e}^{-\Phi(q) y} \mathbb{W}(y+c) \mathrm{d} y$.

Since

$$
\lim _{c \rightarrow \infty}(q-\alpha \Phi(q)) \frac{\int_{-b}^{\infty} \mathrm{e}^{-\Phi(q) y} \mathbb{W}(y+c) \mathrm{d} y}{\mathbb{W}(c)}=\left(\frac{q-\alpha \Phi(q)}{\Phi(q)}\right) \mathrm{e}^{\Phi(q) b},
$$

the result follows, concluding the proof.

Finally, to extract the probability distribution from the Laplace transform, we first modify the expression we have just obtained. Set

$$
v(x)=\mathbb{E}_{x}\left[\mathrm{e}^{-q \int_{0}^{\infty} \mathbf{1}_{\left\{U_{s}<b\right\}} \mathrm{d} s}\right] .
$$

Following the definition of the 0 -scale function and integration by parts, we can write

$$
v(x)=\left(\mathbb{E}\left[X_{1}\right]-\alpha\right)\left[\mathbb{W}(x-b)+\int_{0}^{\infty} \mathrm{e}^{-\Phi(q) y} \mathbb{W}^{\prime}(y+x-b) \mathrm{d} y\right] .
$$

Therefore,

$$
v(x)=\left(\mathbb{E}\left[X_{1}\right]-\alpha\right)\left[\mathbb{W}(x-b)+\int_{0}^{\infty}\left\{\int_{0}^{\infty} \mathrm{e}^{-q s} \mathbb{P}\left(\tau_{y}^{+} \in \mathrm{d} s\right)\right\} \mathbb{W}^{\prime}(y+x-b) \mathrm{d} y\right] .
$$

Note that, by Kendall's identity, we have on $(0, \infty) \times(0, \infty)$

$$
\mathrm{d} y \mathbb{P}\left(\tau_{y}^{+} \in \mathrm{d} r\right)=\frac{y}{r} \mathbb{P}\left(X_{r} \in \mathrm{d} y\right) \mathrm{d} r
$$

and the result follows.

Note that if we set $\alpha=0$ in (i) of the last corollary, we recover Corollary 2(ii) in [16]. Note also that (ii) of the last corollary is a slight improvement over Corollary 2 in [13] in that any initial level $X_{0}=x \in \mathbb{R}$ is considered (the proof is also different). Finally, note that we have obtained a different expression for the density of the total time spent by $U$ below level $b$.

\section{Acknowledgements}

The author thanks the anonymous referee for a very careful reading of the paper.

Funding in support of this work was provided by the Natural Sciences and Engineering Research Council of Canada (NSERC), the Fonds de recherche du Québec - Nature et technologies (FRQNT), and the Institut de finance mathématique de Montréal (IFM2).

\section{References}

[1] Albrecher, H. and Lautscham, V. (2013). From ruin to bankruptcy for compound Poisson surplus processes. Astin Bull. 43, 213-243.

[2] Albrecher, H., Cheung, E. C. K. and Thonhauser, S. (2011). Randomized observation periods for the compound Poisson risk model dividends. Astin Bull. 41, 645-672.

[3] Albrecher, H., Cheung, E. C. K. and Thonhauser, S. (2013). Randomized observation periods for the compound Poisson risk model: the discounted penalty function. Scand. Actuarial J. 2013, 424-452.

[4] Albrecher, H., Gerber, H. U. and Shiu, E. S. W. (2011). The optimal dividend barrier in the gamma-omega model. Europ. Actuarial J. 1, 43-55. 
[5] Czarna, I. ANd Palmowski, Z. (2011). Ruin probability with Parisian delay for a spectrally negative Lévy risk process. J. Appl. Prob. 48, 984-1002.

[6] Egami, M. and YamazaKi, K. (2014). Phase-type fitting of scale functions for spectrally negative Lévy processes. J. Comput. Appl. Math. 264, 1-22.

[7] Gerber, H. U., Shiu, E. S. W. And Yang, H. (2012). The Omega model: from bankruptcy to occupation times in the red. Europ. Actuarial J. 2, 259-272.

[8] Kuznetsov, A., Kyprianou, A. E. And Rivero, V. (2012). The theory of scale functions for spectrally negative Lévy processes. In Lévy Matters II (Lecture Notes Math. 2061), Springer, Heidelberg, pp. 97-186.

[9] Kyprianou, A. E. (2006). Introductory Lectures on Fluctuations of Lévy Processes with Applications. Springer, Berlin.

[10] Kyprianou, A. E. (2013). Gerber-Shiu Risk Theory. Springer, Berlin.

[11] Kyprianou, A. E. And Loeffen, R. L. (2010). Refracted Lévy processes. Ann. Inst. H. Poincaré Prob. Statist. 46, 24-44.

[12] Kyprianou, A. E., Loeffen, R. And PÉrez, J.-L. (2012). Optimal control with absolutely continuous strategies for spectrally negative Lévy processes, J. Appl. Prob. 49, 150-166.

[13] Kyprianou, A. E., Pardo, J. C. And Pérez, J.-L. (2014). Occupation times of refracted Lévy processes. J. Theoret. Prob. 27, 1292-1315.

[14] Landriault, D., Renaud, J.-F. And Zhou, X. (2014). An insurance risk model with Parisian implementation delays. Method. Comput. Appl. Prob. 16, 583-607.

[15] Loeffen, R., Czarna, I. and Palmowski, Z. (2013). Parisian ruin probability for spectrally negative Lévy processes. Bernoulli 19, 599-609.

[16] Loeffen, R., Renaud, J.-F. And Zhou, X. (2014). Occupation times of intervals until first passage times for spectrally negative Lévy processes. Stoch. Process. Appl. 124, 1408-1435. 